treatment in patients with HIV infection and extrapulmonary or MDR tuberculosis. We have no reason to suspect that MDR tuberculosis was unequally distributed in SAPIT study groups, since patients were randomly assigned to treatment and patients with previous tuberculosis at high risk for resistance were evenly distributed among the study groups.

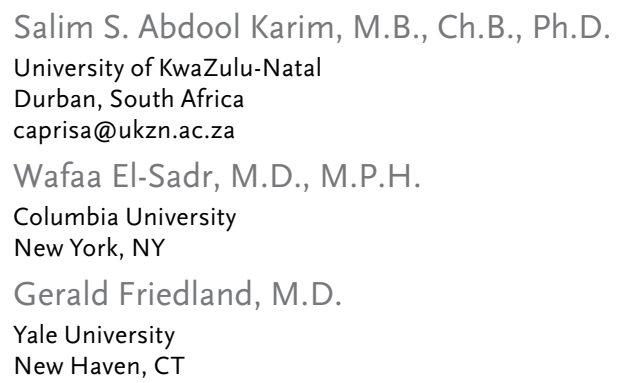

Since publication of their article, the authors report no further potential conflict of interest.

1. Scaling up antiretroviral therapy in resource-limited settings: treatment guidelines for a public health approach. Geneva: World Health Organization, 2003. (Accessed May 18, 2010, at http://whqlibdoc.who.int/publications/2004/9241591552.pdf.)

2. TB/HIV research priorities in resource-limited settings: Report of an expert consultation. Geneva: World Health Organization, 2005. (Accessed May 18, 2010, at http://whqlibdoc.who.int/ hq/2005/WHO_HTM_TB_2005.355.pdf.)

3. Lawn SD, Bekker LG, Miller RF. Immune reconstitution disease associated with mycobacterial infections in HIV-infected individuals receiving antiretrovirals. Lancet Infect Dis 2005;5: 361-73.

4. Müller M, Wandel S, Colebunders R, Attia S, Furrer H, Egger $M$. Immune reconstitution inflammatory syndrome in patients starting antiretroviral therapy for HIV infection: a systematic review and meta-analysis. Lancet Infect Dis 2010;10:251-61.

5. DiGiacinto JL, Chan-Tack KM, Robertson SM, Reynolds KS, Struble KA. Are literature references sufficient for dose recommendations? An FDA case study of efavirenz and rifampin. J Clin Pharmacol 2008;48:518-23.

\title{
Tuberculosis Screening and Diagnosis in People with HIV
}

TO THE EDITOR: Cain et al. (Feb. 25 issue) ${ }^{1}$ conclude that screening for tuberculosis in people with human immunodeficiency virus (HIV) infection should include questions about a combination of symptoms rather than just chronic cough. They suggest that the recently published World Health Organization (WHO) approach ${ }^{2}$ for the diagnosis of tuberculosis among people with HIV has a sensitivity of less than $33 \%$. However, they restrict the WHO approach to the diagnosis of pulmonary tuberculosis and do not consider extrapulmonary tuberculosis. For extrapulmonary tuberculosis, the WHO recommends taking into account other characteristics, such as weight loss, fever, and night sweats. We agree, however, that the use of the three screening criteria (cough of any duration, fever of any duration, and night sweats lasting 3 or more weeks in the preceding 4 weeks) proposed by Cain et al. simplifies the screening and diagnosis of pulmonary and extrapulmonary tuberculosis and will facilitate the implementation of screening and diagnosis at the country level.

Olivier Koole, M.D.

Johan van Griensven, M.D., Ph.D.

Robert Colebunders, M.D., Ph.D.

Institute of Tropical Medicine

Antwerp, Belgium

okoole@itg.be
No potential conflict of interest relevant to this letter was reported.

1. Cain KP, McCarthy KD, Heilig CM, et al. An algorithm for tuberculosis screening and diagnosis in people with HIV. N Engl J Med 2010;362:707-16.

2. Improving the diagnosis and treatment of smear-negative pulmonary and extrapulmonary tuberculosis among adults and adolescents: recommendations for HIV-prevalent and resourceconstrained settings. Geneva: World Health Organization, 2007.

THE AUTHORS REPLY: Koole and colleagues note that our analysis of the WHO approach to tuberculosis screening in people with HIV did not address extrapulmonary tuberculosis. The WHO has guidelines for the diagnosis of extrapulmonary tuberculosis but no explicit guidelines regarding screening. ${ }^{1}$ The guidelines include several symptoms that might prompt a clinician to consider extrapulmonary tuberculosis, but there is no explicit recommendation to screen for them in all patients or to trigger a diagnostic evaluation for tuberculosis when one of them is present. Chronic cough is the only symptom explicitly noted for routine tuberculosis screening. ${ }^{1}$

We reported on symptom screening when patients with all types of tuberculosis were included. We analyzed the data again after excluding the 25 patients who had only extrapulmonary tuberculosis. The sensitivity of cough lasting 2 to 3 weeks 
or more for the detection of culture-proven pulmonary tuberculosis remained low (23 to 34\%). Thus, inclusion of patients with extrapulmonary tuberculosis does not explain the low sensitivity of chronic cough.

In contrast, the symptom combination that we proposed had a sensitivity of $93 \%$ for the detection of pulmonary and extrapulmonary tuberculosis and, therefore, can be used to rule out tuberculosis in all people with HIV infection.

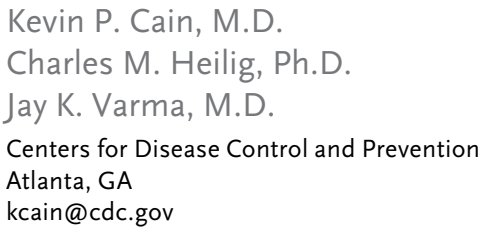

Since publication of their article, the authors report no further potential conflict of interest.

1. Improving the diagnosis and treatment of smear-negative pulmonary and extrapulmonary tuberculosis among adults and adolescents: recommendations for HIV-prevalent and resourceconstrained settings. Geneva: World Health Organization, 2007.

\section{Prophylactic Platelet Transfusions}

TO THE EDITOR: Slichter et al. (Feb. 18 issue) ${ }^{1}$ report on the results of the platelet-dose (PLADO) trial (ClinicalTrials.gov number, NCT00128713). To interpret the results of this study accurately, it would be very important to know the $\mathrm{ABO}$ compatibility between recipients and platelet transfusions. A major ABO incompatibility can decrease the response to a platelet transfusion by between $23 \%$ and $41 \%$ in patients with hematologic cancers. ${ }^{2}$ A platelet transfusion with minor $\mathrm{ABO}$ incompatibility is also associated with a decrease in the post-transfusion increment, perhaps because of immune complexes. ${ }^{3}$ Others have also reported that $\mathrm{ABO}$ compatibility is associated with an increase in the recovery at 1 hour but not at 24 hours. ${ }^{4}$ Moreover, a previous meta-analysis of studies that examined the effect of the transfusion platelet dose on several variables showed that when the studies that guaranteed ABO compatibility of the platelet transfusions were combined, high doses of platelets were associated with a significant increase in the post-transfusion increment as compared with low doses of platelets (weighted mean difference, 23.6×109 platelets per liter; $95 \%$ confidence interval, $18.28 \times 10^{9}$ to $28.92 \times 10^{9}$ per liter; $\left.\mathrm{P}<0.001\right) .^{5}$

\section{Joan Cid, M.D. \\ Ginés Escolar, M.D. \\ Miguel Lozano, M.D. \\ Hospital Clínic \\ Barcelona, Spain \\ jcid.hj23.ics@gencat.cat}

No potential conflict of interest relevant to this letter was reported.

1. Slichter SJ, Kaufman RM, Assmann SF, et al. Dose of prophylactic platelet transfusions and prevention of hemorrhage. N Engl J Med 2010;362:600-13.
2. Lozano M, Cid J. The clinical implications of platelet transfusions associated with $\mathrm{ABO}$ or $\mathrm{Rh}(\mathrm{D})$ incompatibility. Transfus Med Rev 2003;17:57-68.

3. Heal JM, Masel D, Rowe JM, Blumberg N. Circulating immune complexes involving the $\mathrm{ABO}$ system after platelet transfusion. Br J Haematol 1993;85:566-72.

4. Slichter SJ, Davis K, Enright H, et al. Factors affecting posttransfusion platelet increments, platelet refractoriness, and platelet transfusion intervals in thrombocytopenic patients. Blood 2005; 105:4106-14.

5. Cid J, Lozano M. Lower or higher doses for prophylactic platelet transfusions: results of a meta-analysis of randomized controlled trials. Transfusion 2007;47:464-70.

TO THE EDITOR: Slichter et al. report that at doses between $1.1 \times 10^{11}$ and $4.4 \times 10^{11}$ platelets per square meter, the number of platelets in the prophylactic transfusion had no effect on the incidence of bleeding in hospitalized patients undergoing hematopoietic stem-cell transplantation or chemotherapy for hematologic cancers or solid tumors. Although the authors accounted for some confounders (e.g., serum fibrinogen, activated partialthromboplastin time, prothrombin time, and acute promyelocytic leukemia), they did not account for other relevant risk factors for bleeding, such as mucosal or vascular infiltration by solid tumors, chemotherapy-induced gastrointestinal mucositis, graft-versus-host disease, ${ }^{1}$ hemorrhagic cystitis, ${ }^{2}$ and veno-occlusive disease after allogeneic hematopoietic stem-cell transplantation. ${ }^{3}$

Finally, although they did not directly account for bleeding events, the number of required platelet transfusions could be related to post-transfusion increments, which are known to be higher when platelets are stored for less than 6 days. ${ }^{4}$

Daniele Focosi, M.D.

University of Pisa

Pisa, Italy

dfocosi@tin.it 